\title{
ANGIO TC EN LA IDENTIFICACIÓN PREOPERATORIA DE VASOS PERFORANTES*
}

\author{
Drs. Patricio Andrades C. ${ }^{1}$, María Elsa Calderón G. ${ }^{1}$, \\ Patricio Palaviccino R. ${ }^{1}$, Stefan Danilla E. ${ }^{1}$, Cristian Erazo C. ${ }^{1}$, \\ Susana Benítez S. ${ }^{1}$, Sergio Reyes J. ${ }^{1}$, Sergio Sepúlveda P. ${ }^{1}$ \\ ${ }^{1}$ Departamento de Cirugía Hospital Clínico Universidad de Chile. \\ Santiago, Chile.
}

\begin{abstract}
\section{CT Angiography in preoperative identification of perforator vessels}

Introduction: Perforator flaps have become a coverage option validated and increasingly used in reconstructive surgery. These flaps require preoperative identification and intraoperative dissection of the perforator vessels. The aim of this study was to evaluate the use of Angio CT in the preoperative assessment of perforating vessels and its correlation with intraoperative findings. Material and Methods: We retrospectively reviewed all perforator flaps performed at the Division of Plastic Surgery, University of Chile Clinical Hospital between June 2009 and June 2012. All patients were asked for an Angio CT according to the protocol of the University of Gent (Belgium). Data points were used and a specific coordinate system to locate the point at which the perforator vessel fenestrate the deep fascia. The information provided in the Angio CT was compared with surgical findings. Results: A total of 41 patients underwent perforator flap planning during the study period. 40 underwent surgery: 18 women and 22 men, with a mean age of $46.6 \pm 5.7$ years. Flaps were performed as follows: anterolateral thigh $(\mathrm{ALT})=14$, inferior epigastric $(\mathrm{DIEP})=18$, superior gluteal $(\mathrm{SGAP})=5$, posterior tibial $(\mathrm{PTAP})=4$ and thoracodorsal $(\mathrm{TDAP})=2$. In all cases the Angio CT identified one or more perforator vessel with a $100 \%$ concordance with intraoperative findings. Conclusions: CT Angio is a test with high performance in preoperative characterization of perforator vessels. It is a helpful tool that must be considered whenever planning this type of flaps.
\end{abstract}

Key words: Perforator vessels, flaps, Angio CT, reconstructive surgery.

\section{Resumen}

Introducción: Los colgajos de perforantes se han convertido en una opción de cobertura válida cada vez más utilizada en cirugía reconstructiva. Se caracterizan porque requieren de la identificación preoperatoria y disección intraoperatoria del vaso perforante. El objetivo del presente trabajo fue evaluar el Angio TC en el estudio preoperatorio de los vasos perforantes y su correlación con los hallazgos intraoperatorios. Material

*Recibido el 10 de junio de 2013 y aceptado para publicación el 4 de septiembre de 2013.

Los autores no refieren conflictos de interés.

Correspondencia: Dr. Patricio Andrades C.

Santos Dumont No 999, Santiago, Chile.

pandrades@uchile.cl 
y Métodos: Análisis retrospectivo de todos los colgajos de perforantes realizados en la Unidad de Cirugía Plástica del Hospital Clínico de la Universidad de Chile entre junio de 2009 y junio de 2012. A todos los pacientes se les solicitó un Angio TC según el protocolo de la Universidad de Gent (Bélgica). Se utilizaron puntos de referencias específicos y un sistema de coordenadas para localizar el punto en el cual la perforante cruza la fascia superficial. La información entregada por el Angio TC se comparó con los hallazgos quirúrgicos. Resultados: A un total de 41 pacientes se les realizó Angio TC para la planificación de un colgajo de perforante durante el período en estudio, 40 fueron operados: 18 mujeres y 22 hombres, edad promedio de 46,6 \pm 5,7 años. Se realizaron los siguientes colgajos: Anterolateral de Muslo (ALT) = 14, Epigástrica Inferior $(\mathrm{DIEP})=18$, Glútea Superior $(\mathrm{SGAP})=5$, Tibial Posterior $(\mathrm{PTAP})=4$ y Toracodorsal $($ TDAP $)=2$. En todos ellos el Angio TC ubicó una o varias perforantes en forma precisa y $100 \%$ concordante con los hallazgos intraoperatorios. Conclusiones: El Angio TC es un examen con alto rendimiento en la identificación preoperatoria del vaso perforante y su trayecto. Es una herramienta de gran ayuda al momento de planificar la realización de este tipo de colgajos.

Palabras clave: Vasos perforantes, colgajo, AngioTC, cirugía reconstructiva.

\section{Introducción}

Los colgajos perforantes se definen según el Consenso de Gent del año 2003, como una isla de piel y tejido celular subcutáneo que es irrigado por un determinado vaso perforante, independientemente de si se trata de un vaso directo (septocutáneo) o indirecto (musculocutáneo), que se diseca desde el músculo pero sin desfuncionalizarlo ${ }^{1}$. Hallock define a una perforante como cualquier vaso que entra al plano superficial a través de una fenestración definida en la fascia, independientemente de su origen²

Descritos inicialmente en el año 1988 por $\mathrm{Kroll}^{3}$ y estudiados posteriormente por Koshima ${ }^{4}$ y Hallock $^{2}$, los colgajos perforantes se han convertido durante los últimos años en una herramienta muy útil para la cobertura de defectos de partes blandas y en algunas regiones del cuerpo son considerados la primera elección ${ }^{5}$. Dentro de sus ventajas están la disminución de la morbilidad de la zona dadora, ya que preserva la inervación, irrigación y funcionalidad del músculo donante; disminuyen el dolor postoperatorio con una consecuente mejor rehabilitación; ofrecen también pedículos vasculares más largos, y una mínima secuela estética. Las dos principales diferencias entre los colgajos perforantes y el resto de los colgajos son, que los primeros requieren de la identificación preoperatoria y la disección intraoperatoria del vaso perforante ${ }^{6}$.

En sus inicios, las perforantes se identificaban en el intraoperatorio a través de parámetros de referencia anatómicos, basados en descripciones de expertos lo que representa un amplio margen de error. Dentro de las "sorpresas" intraoperatorias que se podían encontrar estaban la ausencia del vaso, el origen distinto, el menor diámetro, ramificación atípica y pedículos insuficientes. Además, esta incertidumbre hacía que la búsqueda del vaso perforante se realizara bajo presión y tomara largo tiempo. Por este motivo se han utilizado múltiples métodos de imágenes para la identificación de los vasos perforantes con resultados variables. Entre ellos, el que ha demostrado mayor utilidad es la Tomografía axial computada con angiografía (Angio TC) ) $^{7-9}$.

En este contexto, el objetivo del presente trabajo fue evaluar el uso del Angio TC como método de estudio de vasos perforantes, sistematizar los puntos de referencias para su identificación preoperatoria y evaluar su correlación con los hallazgos intraoperatorios.

\section{Material y Método}

Se revisaron en forma retrospectiva todos los colgajos de perforantes libres y pediculados realizados en la Unidad de Cirugía Plástica del Hospital Clínico de la Universidad de Chile entre junio de 2009 y junio de 2012. A todos los pacientes se les solicitó una Angio TC preoperatoria (1 semana antes de la cirugía), según el protocolo de la Universidad de Gent (Bélgica) modificado para la inyección de medio de contraste y adquisición de imágenes ${ }^{10}$. Los detalles de dicho protocolo aparecen resumidos en la Tabla 1. Utilizando el Angio TC, se identifica la o las perforantes en el lugar de fenestración de la fascia profunda. Luego este punto de fenestración es llevado a la superficie cutánea utilizando las imágenes tridimensionales obtenidas del escáner. En conjunto con los radiólogos, se estandarizan puntos de referencia y un sistema de coordenadas para cada colgajo en particular. Finalmente, utilizando este sistema de coordenadas el radiólogo puede informar al cirujano de la localización exacta de la perforante, para que este pueda planificar adecuadamente su cirugía. La información entregada por el Angio TC fue comparada con los hallazgos intraoperatorios.

Se aplicó la misma metodología para los siguientes tipos de colgajos de perforantes: Colgajo de arteria Epigástrica Inferior Profunda (DIEP), 
Tabla 1. Protocolo angio TC para el estudio vascular de colgajos perforantes

\begin{tabular}{|ll|}
\hline Scanner & Siemmens Somaton Sensation 64 \\
Rango & Depende de zona anatómica (abdomen, tórax, extremidades inferiores, etc) \\
\hline Dirección & Intravenoso, $5 \mathrm{ml} / \mathrm{seg}$ en vena antecubital \\
\hline Colimación del Corte & Céfalo-caudal \\
\hline Grosor del Corte & $1,5 \mathrm{~mm}$ \\
\hline Pitch (rotación de alimentación) & $3 \mathrm{~mm}$ \\
\hline Tiempo de rotación & 0,7 \\
\hline Mas & $0,5 \mathrm{seg}$ \\
\hline KV & $200 \mathrm{~mA}$ \\
Adquisición & 120 \\
\hline Filtro de Reconstrucción & $64 \times 0,6$ \\
\hline
\end{tabular}

Tabla 2. Características generales y puntos de referencia radiológicos para los 5 colgajos de perforantes incluidos en el estudio

\begin{tabular}{|c|c|c|c|c|}
\hline Colgajo & Arteria fuente & Pedículo & $\begin{array}{l}\text { Dimensión } \\
\text { promedio }\end{array}$ & Puntos de Referencia para Angio TC \\
\hline DIEP & A. Epigástrica profunda inferior & $\begin{array}{l}\text { L: } 8-20 \mathrm{~cm} \\
\text { D: } 3,3 \mathrm{~mm}\end{array}$ & $18 \times 12 \mathrm{~cm}$ & Ombligo \\
\hline ALT & $\begin{array}{l}\text { Rama descendente } \\
\text { A. Circunfleja femoral lateral }\end{array}$ & $\begin{array}{l}\mathrm{L}: 8-16 \mathrm{~cm} \\
\mathrm{D}: 2,1 \mathrm{~mm}\end{array}$ & $30 \times 9 \mathrm{~cm}$ & $\begin{array}{l}\text { Punto medio de una línea que une la } \\
\text { espina ilíaca anterosuperior y el borde } \\
\text { lateral de la rótula }\end{array}$ \\
\hline PTAP & A. Tibial posterior & $\begin{array}{l}\mathrm{L}: 5-15 \mathrm{~cm} \\
\mathrm{D}: 2-3 \mathrm{~mm}\end{array}$ & $8 \times 16 \mathrm{~cm}$ & $\begin{array}{l}\text { Punto sobre una línea en el borde medial } \\
\text { de la tibia a nivel del maléolo medial }\end{array}$ \\
\hline TDAP & A. Toracodorsal & $\begin{array}{l}\mathrm{L}: 13 \mathrm{~cm} \\
\mathrm{D}: 2,2 \mathrm{~mm}\end{array}$ & $11 \times 7 \mathrm{~cm}$ & $\begin{array}{l}\text { Punto de intersección de una línea hori- } \\
\text { zontal que pasa a través del xifoides con } \\
\text { la línea axilar media }\end{array}$ \\
\hline SGAP & A. Glútea superior & $\begin{array}{l}\text { L: } 3-8 \mathrm{~cm} \\
\text { D: } 1,8-3 \mathrm{~mm}\end{array}$ & $10 \times 15 \mathrm{~cm}$ & Coxis \\
\hline
\end{tabular}

DIEP: colgajo perforante de arteria epigástrica profunda inferior; ALT: colgajo anterolateral de muslo; PTAP: colgajo perforante de arteria tibial posterior; SGAP: colgajo perforante de arteria glútea superior.

Colgajo Anterolateral de Muslo (ALT), Colgajo de arteria Tibial Posterior (PTAP), Colgajo de arteria Toracodorsal (TDAP) y Colgajo de arteria Glútea Superior (SGAP). Los puntos de referencia y el punto cero del sistema de coordenadas para cada uno de estos colgajos se describen en la Tabla 2. Para el colgajo DIEP el punto 0 es el ombligo; para el Colgajo ALT es el punto medio de una línea que une la espina ilíaca anterosuperior y el borde lateral de la rótula; para el Colgajo PTAP es el punto sobre una línea en el borde medial de la tibia a nivel del maléolo medial; para el Colgajo TDAP es el punto de intersección de una línea horizontal que pasa a través del xifoides con la línea axilar media; y para el Colgajo SGAP es el coxis.

\section{Resultados}

A un total de 41 pacientes se les solicitó Angio TC para la planificación de un colgajo de perforante durante el período en estudio. 40 fueron operados. Hubo 18 mujeres y 22 hombres, con una edad promedio de 46,6 $\pm 5,7$ años. Se realizaron los siguientes colgajos: Anterolateral de Muslo $(\mathrm{ALT})=14$, Epigástrica Inferior $(\mathrm{DIEP})=18$, Glútea Superior 
$(\mathrm{SGAP})=5$, Tibial Posterior $(\mathrm{PTAP})=4 \mathrm{y}$ Toracodorsal $($ TDAP $)=2$. En todos ellos el Angio TC ubicó una o varias perforantes en forma precisa y $100 \%$ concordante con los hallazgos intraoperatorios. Además el Angio TC permitió caracterizar la perforante en su trayecto pre-fascial, intramuscular y post-fascial. Solo una paciente presentó un "inciden- taloma" (cáncer de mama identificado en el estudio de perforante toracodorsal) que finalmente impidió la realización del colgajo.

El esquema de coordenadas, las imágenes preoperatorias, la marcación y el hallazgo intraoperatorio de algunos casos clínicos pueden observarse en las Figuras 1 a 5.
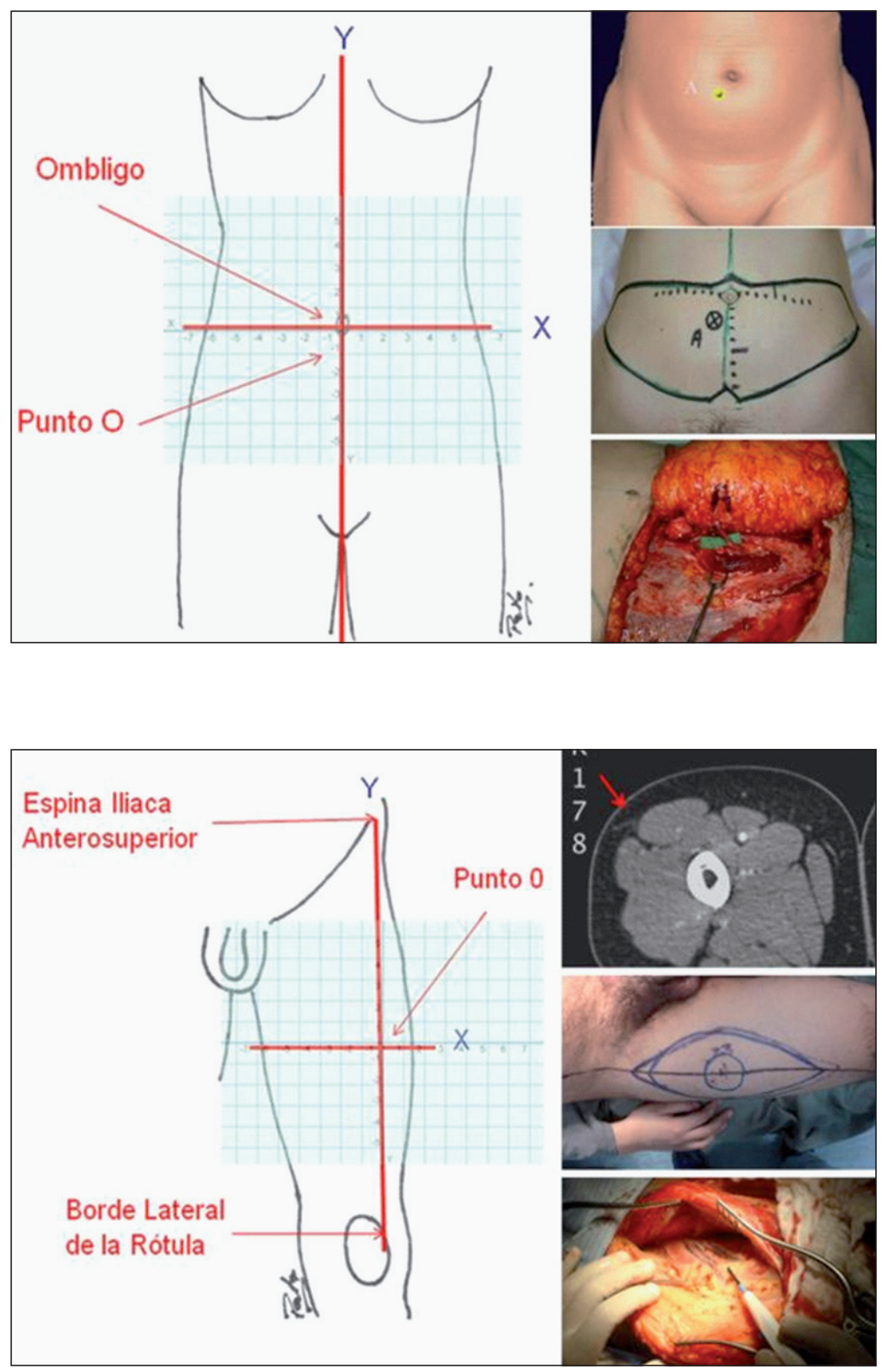

Figura 1. El punto de referencia para el Colgajo DIEP es el ombligo. Este punto sirve de referencia como el punto 0 para generar las coordenadas $\mathrm{X}$ e Y. A la derecha las coordenadas $\mathrm{X}=-1,6$ e $\mathrm{Y}=-3-5$ informadas en el Angio TC son aplicadas a la paciente con perfecta concordancia intraoperatoria.
Figura 2. Los puntos de referencia para el Colgajo ALT son la Espina Iliaca Anterosuperior y el borde lateral de la rótula. El punto 0 se obtiene a nivel del punto medio de la línea que une ambos puntos de referencia. A la derecha se informa punto media a $23,6 \mathrm{~cm}$ de la EIAS con coordenadas $\mathrm{Y}=1,2$ y $\mathrm{X}=-1,7$ y perfecta concordancia intraoperatoria de perforante septocutánea. 


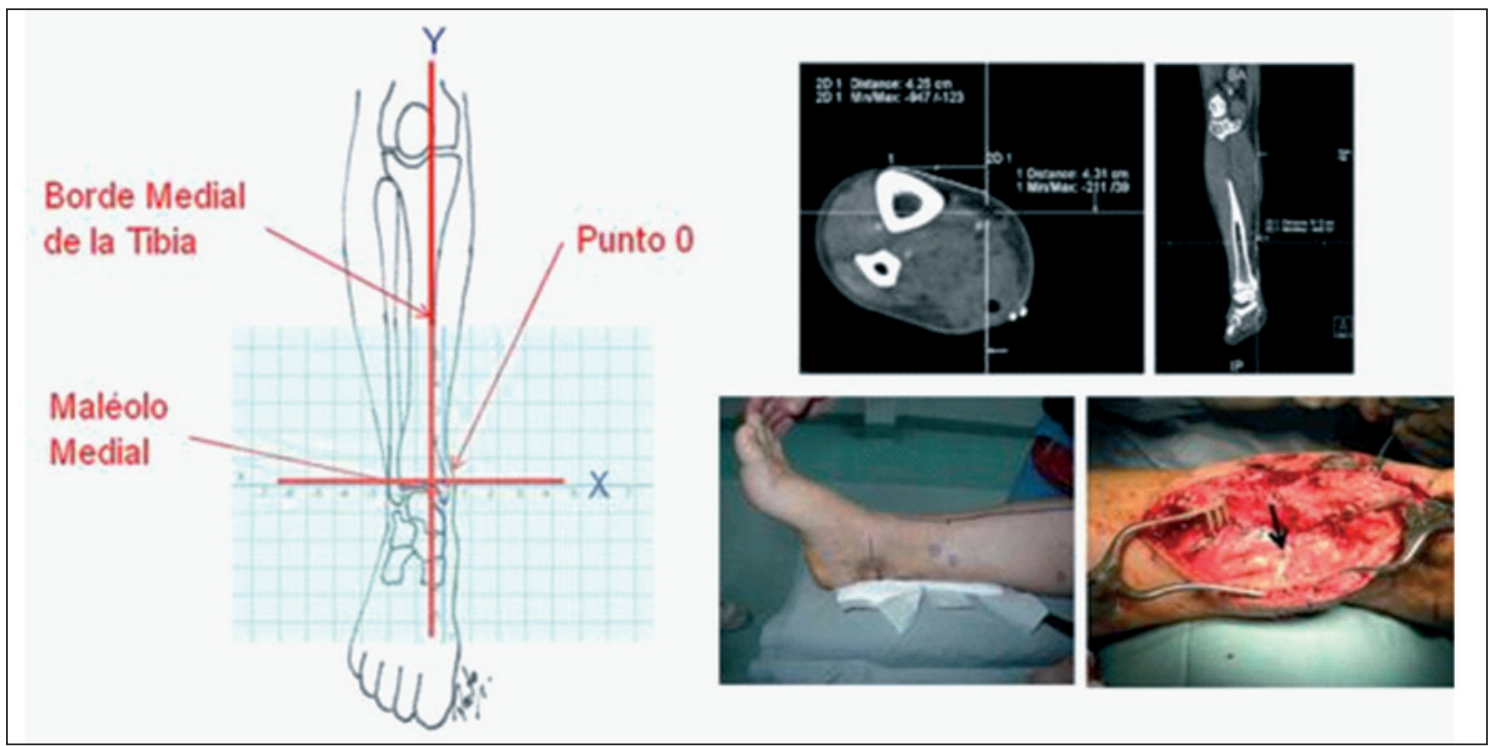

Figura 3. Los puntos de referencia para el Colgajo PTAP son la del borde medial de la Tibia y el Maléolo medial. El punto 0 se obtiene en la intersección de ambas líneas. A la derecha se informan las coordenadas $\mathrm{Y}=8,1$ y $\mathrm{X}=2,3$ y perfecta concordancia intraoperatoria.

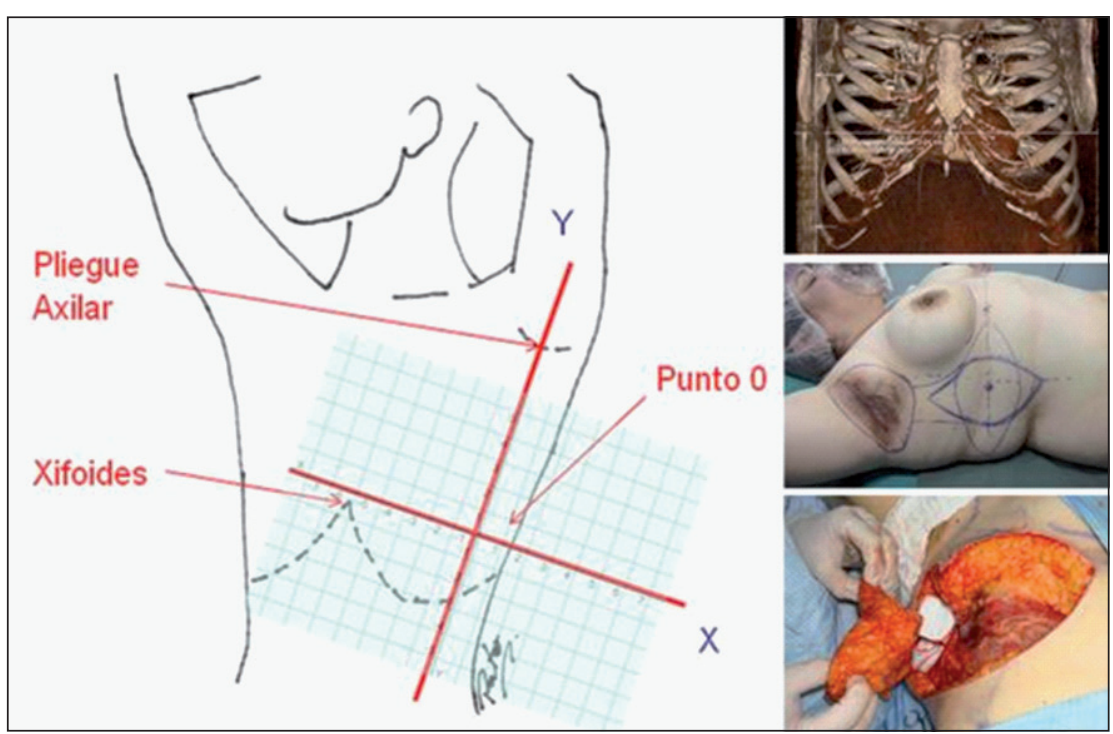

Figura 4. Los puntos de referencia para el Colgajo PTAP son el pliegue axiilar y el apéndice xifoides. El punto 0 se obtiene de la intersección entre la perpendicular al pliegue axilar y la línea transversa a nivel del xifoides. A la derecha se informa punto de intersección a $22,5 \mathrm{~cm}$ de la axila con coordenadas $\mathrm{Y}=6$ y $X=0,5$ y perfecta concordancia intraoperatoria.

\section{Discusión}

En la actualidad existe creciente evidencia apoyando el uso de algún tipo de imágenes para la caracterización preoperatoria de vasos perforantes en cirugía reconstructiva ${ }^{11}$. Entre las modalidades más utilizadas están: el Doppler de bolsillo ${ }^{12}$,Eco-Doppler $^{13}$, Tomografía Computarizada ${ }^{9}$, y Resonancia Nuclear Magnética ${ }^{14}$. El Doppler de bolsillo tiene una alta sensibilidad, pero baja especificidad y puede consumir mucho tiempo ${ }^{15}$. El Eco-Doppler aporta muchísima información en especial la hemodinámica, pero es operador dependiente y no permite tener imágenes anatómicas para el intraoperatorio ${ }^{13}$. Finalmente, la Angioresonancia es un excelente examen que no irradia al paciente, pero no está ampliamente disponible y sus costos son mucho más elevados ${ }^{16,17}$. Cada modalidad presenta sus ventajas y desventajas; 


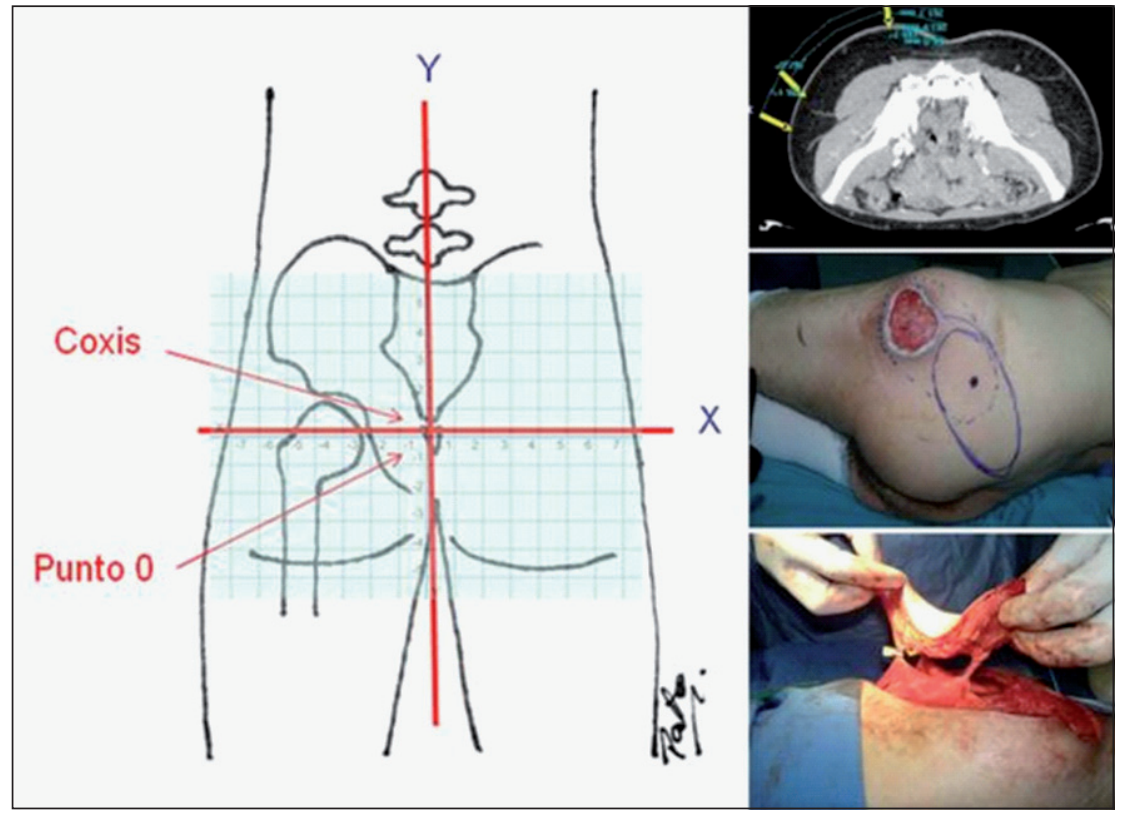

Figura 5. El punto de referencia para el Colgajo SGAP es el Coxis. Este punto sirve de referencia como el punto 0 para generar las coordenadas $\mathrm{X}$ e $\mathrm{Y}$. A la derecha las coordenadas $\mathrm{X}=-17,8$ e $\mathrm{Y}=2,5$ informadas en el Angio TC son aplicadas al paciente con perfecta concordancia intraoperatoria.

Tabla 3. Análisis comparativo de las diferentes modalidades utilizadas para el estudio vascular preoperatorio en colgajos perforantes

\begin{tabular}{|lllll|}
\hline \multirow{2}{*}{ Ventajas } & Doppler & ECO Doppler & Angioresonancia & Angio TC \\
& Portable & Alta Sensibilidad & No irradia & Alto rendimiento \\
& Barato & Hemodinamia & Alto rendimiento & Mapeo tridimensional \\
& & & Imágenes intraop. & Visualiza otras estructuras \\
Desventajas & & Visualiza otras estructuras & Imágenes intraop. \\
& Información limitada & Poca Especificidad & Baja disponibilidad & Irradiación \\
& Baja Sensibilidad y & Operador dependiente & Toma compleja & No evalúa hemodinamia \\
& Especificidad & Consume tiempo & Alto costo & \\
& Consume tiempo & & No evalúa hemodinamia & \\
\hline
\end{tabular}

sin embargo, en la actualidad la Angiografía por Tomografía Computarizada (Angio TC) es considerado el patrón de oro para la detección y caracterización de los vasos perforantes (Tabla 3).

En nuestro trabajo encontramos $100 \%$ de concordancia en cuanto a la ubicación de la perforante informada por el radiólogo y los hallazgos intraoperatorios. Estos resultados son concordantes con los publicados en la literatura extranjera ${ }^{18}$. Burgos ${ }^{8}$ y Masia $^{9}$, reportan por primera vez, el mismo año (2006) y en forma independiente el uso de este estudio imagenológico con un $100 \%$ de sensibilidad y especificidad. Casey et al ${ }^{19}$, el año 2009, publican sus resultados en 219 pacientes utilizando el Angio TC preoperatoria para marcar perforantes de colgajo
DIEP. Ellos no solo informan un altísimo rendimiento de la técnica sino también disminución del tiempo operatorio, de las complicaciones en la zona dadora y una mejora en la curva de aprendizaje en forma estadísticamente significativa. Phillips et al ${ }^{10}$, aportan incluso mayor importancia a la técnica, ya que demuestran que la Angio TC no solo informa el punto en que la perforante atraviesa la fascia, sino que también da información acerca de su origen, trayecto pre-muscular, intra-muscular, y arborización post-fascial en el tejido celular subcutáneo. Este último punto es muy importante, y nosotros aunque no lo cuantificamos, también pudimos observarlo durante nuestro trabajo. Es importante ya que permite en el preoperatorio planificar en forma detallada 
la cirugía, seleccionar el vaso dependiendo de su diámetro, localización y trayecto, planificar un plan "B" en caso de fallo y ahorrar tiempo. Pratt et al, en una reciente revisión sistemática de la literatura encuentra que de todas las modalidades de imágenes utilizadas para el estudio preoperatorio de los vasos perforantes, existe una creciente evidencia nivel $2 \mathrm{~b}$ apoyando el uso del Angio $\mathrm{TC}^{11}$.

Por todo lo anterior, para la programación, confección adecuada del colgajo y una cirugía reconstructiva exitosa, se hace evidente la necesidad de un apoyo imagenológico preciso. A pesar de que el Angio TC ha resultado ser una excelente técnica, puede tener problemas que en la mayoría de los casos pueden prevenirse. El protocolo para la realización del Angio TC es fundamental, especialmente lo que se refiere a la velocidad de inyección del medio de contraste, tiempo de espera retardado para la toma de imágenes, y definición de las imágenes propiamente tal (espacio entre cortes y resolución) ${ }^{10}$. De no seguir exactamente los pasos, las imágenes obtenidas pueden ser de mala calidad y llevar a error. Es también muy importante tener una adecuada comunicación con el radiólogo para acordar puntos de referencias claros y un lenguaje en común que permita la más alta precisión. Finalmente, la Angio TC no solo permite ver los vasos, sino también otras estructuras corporales relacionadas y no tan relacionadas con la cirugía. Se pueden analizar los vasos receptores y las estructuras musculares subyacentes, como también órganos más profundos. Por ejemplo, en una de nuestras pacientes no se pudo realizar la cirugía ya que el escáner detectó incidentalmente un cáncer en la mama contralateral que determinó un cambio absoluto de planes. Todos estos elementos se deben tener en cuenta para optimizar la técnica y obtener los mejores resultados.

En resumen, la Angio TC es un examen con alto rendimiento en la identificación preoperatoria de vasos perforantes y de su trayecto. Analizando las distintas alternativas de apoyo imagenológico y evaluando los métodos usualmente utilizados para la identificación de los vasos perforantes, se hacen evidentes las razones para elegirlo como método diagnóstico de elección a la hora de programar una cirugía reconstructiva con un colgajo de perforantes, considerando que nuestros pacientes suelen ser casos complejos, donde no contamos con muchas alternativas para cobertura $\mathrm{y}$, por lo tanto, no hay mucho margen de error. La anatomía vascular suele ser muy distinta de la descripción habitual y entre un paciente y otro; pequeñas variaciones en ella pueden significar el fracaso de la cirugía y la pérdida de una potencial zona dadora. El Angio TC ha llegado a cumplir un rol trascendental en el estudio preoperatorio; no solo por el beneficio de disminuir el tiempo quirúrgico, ser no invasivo, operador independiente, rápido y reproducible, capaz de evaluar milimétricamente la localización, origen, diámetros y trayectos de los vasos tanto de zonas dadoras como de zonas receptoras con una alta sensibilidad y especificidad, facilitando la planificación de la cirugía y evitando tomar decisiones intraoperatorias de último minuto que pueden no ser la mejor solución para el paciente $^{20}$. Por estas razones, es una herramienta que debiera considerarse siempre al momento de realizar este tipo de cirugías.

\section{Referencias}

1. Blondeel P, Van L, Monstrey S, Hamdi M, Matton G, Allen R, et al. The "Gent" consensus on perforator flap terminology: preliminary definitions. Plast Reconstr Surg. 2003;112:1378-83.

2. Hallock G. Direct and indirect perforator flaps: the history and the controversy. Plast Reconstr Surg. 2003;111:855-65.

3. Kroll S, Rosenfield L. Perforator-based flaps for low posterior midline defects. Plast Reconstr Surg. 1988;81:561-6.

4. Koshima I, Soeda S. Inferior epigastric artery skin flaps without rectus abdominis muscle. Br J Plast Surg. 1989;42:645-8.

5. Saint-Cyr M, Schaverien M, Rohrich R. Perforator flaps: history, controversies, physiology, anatomy, and use in reconstruction. Plast Reconstr Surg. 2009;123:132-45.

6. Wei F, Celik N. Perforator flap entity. Clin Plast Surg. 2003;30:325-9.

7. Rozen W, Phillips TJ, Ashton M, Stella D, Gibson R, Taylor G. Preoperative imaging for DIEA perforator flaps: a comparative study of computed tomographic angiography and doppler ultrasound. Plast Reconstr Surg. 2008;121:1-8.

8. Alonso-Burgos A, García-Tutor E, Bastarrika G, Cano D, Martínez-Cuesta A, Piña L. Preoperative planning of deep inferior epigastric artery perforator flap reconstruction with multislice-CT angiography: imaging findings and initial experience. J Plast Reconstr Aesthet Surg. 2006;59:585-93.

9. Masia J, Clavero J, Larranaga J, Alomar X, Pons G, Serret P. Multidetector-row computed tomography in the planning of abdominal perforator flaps. J Plast Reconstr Aesthet Surg. 2006;59:594-9.

10. Phillips T, Stella D, Rozen W, Ashton M, Taylor G. Abdominal wall CT angiography: a detailed account of a newly established preoperative imaging technique. Radiology 2008;249:32-44.

11. Pratt G, Rozen W, Chubb D, Ashton M, Alonso-Burgos A, Whitaker I. Preoperative imaging for perforator flaps in reconstructive surgery: a systematic review of the evidence for current techniques. Ann Plast Surg. 2012;69:3-9. 
12. Hallock G. Doppler sonography and color duplex imaging for planning a perforator flap. Clin Plast Surg. 2003;30:347-57.

13. Hallock G. Acoustic Doppler sonography, color duplex ultrasound, and laser Doppler flowmetry as tools for successful autologous breast reconstruction. Clin Plast Surg. 2011;38:203-11.

14. Polanco D, Fernández-Cañamaque F, Ortega I, Castillo E. Utilidad de la angioresonancia en la planificacion de colgajos libres abdominales DIEP, SIEA y TRAM. Rev Chil Cir. 2010;62:449-53.

15. Hallock G. Attributes and shortcomings of acoustic Doppler sonography in identifying perforators for flaps from the lower extremity. J Reconstr Microsurg. 2009;25:377-81.

16. Schaverien M, Ludman C, Neil-Dwyer J, McCulley S. Contrast-enhanced magnetic resonance angiography for preoperative imaging of deep inferior epigastric artery perforator flaps: advantages and disadvantages compared with computed tomography angiography: a United Kingdom perspective. Ann Plast Surg. 2011;67:671-4.

17. Masia J, Navarro C, Clavero J, Alomar X. Noncontrast magnetic resonance imaging for preoperative perforator mapping. Clin Plast Surg. 2011;38:253-61.

18. Aubry S, Pauchot J, Kastler A, Laurent O, Tropet Y, Runge M. Preoperative imaging in the planning of deep inferior epigastric artery perforator flap surgery. Skeletal Radiol. 2013;42:319-27.

19. Casey W, Chew R, Rebecca A, Smith A, Collins J, Pockaj BA. Advantages of preoperative computed tomography in deep inferior epigastric artery perforator flap breast reconstruction. Plast Reconstr Surg. 2009;123:1148-55.

20. Basu A, Sharma R. "Image-Guided" Perforator Flaps versus "Free-Style" Perforator Flaps: Where Is the Evidence? Plast Reconstr Surg. 2013;131:444-5. 